\title{
Las herramientas de evaluación en las empresas de inserción según las fases del itinerario: un ejemplo en el País Vasco
}

\author{
Juan José Zacarés González \\ Departamento de Psicología Evolutiva y de la Educación, Universitat de València \\ <Juan.Jose.Zacares@uv.es>
}

\section{Lucía I. Llinares Insa}

Departamento de Psicología Social, Universitat de València

\section{Ana I. Córdoba Iñesta}

Departamento de Psicología Evolutiva y de la Educación, Universitat de València

\begin{abstract}
Artikulu honetan aurkezten da analisi bat gizarteratzeko enpresek erabilitako ebaluaziorako tresnak ezagutzeko. Ikerketaren xedea da panoramika adierazgarri bat aurkeztea Estatu espainiarrean erabilitako ebaluaziorako tresnen inguruan. Gainera, artikuluak jasotzen ditu Euskadiko araudiaren gai partikular batzuek. Xedea da kritikoki ebaluatzea erabilitako tresna horien betekizun-maila ezagutzeko. Bertan ondorio gisara azaltzen dira ebaluazioaren arlo honetarako zein alderdi diren gizarteratzeko enpresa horiek erabili behar dituztenak, eta iradokitzen dira zenbait bide tresna horiek eta berauen erabileran hobetu ahal izateko.
\end{abstract}

\section{HITZ-GAKOAK:}

ebaluaziorako tresnak, gizarteratzea eta laneratzea, gizarteratzeko ibilbideak, enplegagarritasuna, gizarteratzeko enpresa.
Este artículo presenta un primer nivel de análisis de las herramientas evaluativas que utilizan las empresas de inserción en cada una de las fases de los itinerarios de inserción. Se trata de ofrecer una visión panorámica a partir de una muestra representativa de instrumentos de evaluación empleados por empresas de inserción de todo el Estado español, en el que también se detallan algunas particularidades normativas para el caso del País Vasco. El objetivo es valorar críticamente el grado en que estas herramientas están sirviendo a los fines de cada una de las fases del proceso seguido por los trabajadores de inserción. Se concluye señalando los aspectos clave que las empresas de inserción han de considerar en esta dimensión evaluativa, y apuntando posibles vías de mejora en las herramientas y en el uso que se hace de ellas.

\section{PALABRAS ClaVe:}

herramientas de evaluación, inserción sociolaboral, itinerarios de inserción, empleabilidad, empresa de inserción. 


\section{Introducción: la evaluación a lo largo de las fases del itinerario de inserción}

Las empresas de inserción son una de las iniciativas sociales de la lucha contra la exclusión (Ley 12/1998, de 22 de mayo). En el intento de insertar sociolaboralmente a quienes carecen de recursos personales, sociales o económicos suficientes para desarrollar una vida independiente, las empresas de inserción tienen como uno de los fines prioritarios construir itinerarios de inserción con el trabajador que sirvan de estructura de apoyo a su empleabilidad. Los aspectos que se consideran en este sentido son de diversa naturaleza: la presencia o ausencia de ciertos indicadores de empleabilidad en los instrumentos utilizados y el nivel de operativización/estructuración de dichos instrumentos, el tipo de factores recogidos más frecuentemente en los instrumentos o el uso que se hace de estas herramientas evaluativas, entre otros. Todas estas dimensiones nos informan del concepto de empleabilidad que se maneja en una empresa de inserción determinada. El análisis de los instrumentos de valoración es, por tanto, una de las vías para acceder a los criterios de empleabilidad subyacentes sobre los cuales podremos realizar la posterior reflexión crítica (Zacarés, Córdoba y Llinares, 2011). Presentamos en este artículo el primer nivel de análisis de las herramientas de evaluación que emplean las empresas de inserción del Estado español, junto a un comentario particular para el caso del País Vasco. Un segundo nivel analítico permite profundizar en las dimensiones de empleabilidad subyacentes, tal y como se expone en otro trabajo (Llinares, Córdoba y Zacarés, 2012).

Dicho análisis tiene como telón de fondo las fases generales del itinerario de inserción, a saber, las de acogida, seguimiento, y transición o tránsito al mercado laboral ordinario. Se hace necesario mencionar los objetivos planteados en cada una, ya que en relación a ellos se han concebido los diversos instrumentos que posteriormente se examinan (González y Marhuenda, 2008).

\subsection{Primera fase: acogida (también denominada fase de incorporación o de evaluación inicial)}

La de acogida es una fase de comunicación y conocimiento de expectativas mutuas entre el trabajador y la empresa. La empresa de inserción, a través de los técnicos y profesionales ubicados en esta fase, transmite al trabajador los objetivos como empresa de inserción, qué caracteriza al puesto de trabajo y el tiempo previsible de permanencia en ella, sin perder de vista la meta final de acceso al mercado laboral ordinario una vez recorrido el itinerario establecido. Por su parte, el trabajador expone la situación vital desde la que accede a la empresa y confronta las disposiciones, motivaciones y capacidades que puede poner en juego mientras permanezca en la empresa.

En esta fase se producen dos hitos importantes para todo el proceso:
- La valoración inicial, dentro de la cual se enmarca el diagnóstico de empleabilidad: a partir de diversos procedimientos de evaluación (entrevistas, dinámicas grupales, aplicación de cuestionarios y pruebas estandarizadas, observación) se pretende un doble objetivo: a) valorar capacidades generales y específicas vinculadas a la empleabilidad (de naturaleza cognitiva, social o personal) que forman parte del bagaje biográfico de la persona, especialmente de su trayectoria sociolaboral; y b) ubicar actitudinalmente al futuro trabajador (Orteu, 2007), es decir, captar cómo la persona define su situación y a qué la atribuye, qué conoce y desconoce del mercado laboral, qué está dispuesto a hacer, cómo se representa la oferta de la empresa de inserción y cómo define la demanda que ésta le hace.

- La elaboración de un itinerario de inserción personalizado: de la evaluación anterior surge una propuesta de intervención que trata de incidir en las carencias detectadas.

Esta propuesta se concreta en el diseño, junto con el trabajador, de su propio itinerario de inserción. González y Marhuenda (2008) sintetizan sus notas características:

- Se trata de un acuerdo conocido y reconocido por ambas partes.

- Es un plan de trabajo como articulación de objetivos y acciones, tanto educativas como no educativas, que ha de realizar quien recorre el itinerario.

- Es un horizonte valioso a medio y largo plazo, que proyecta a la persona hacia un futuro y que funciona "como invitación a que las personas tomen decisiones, a que se conviertan en protagonistas de sus vidas y dejen de estar sujetos a las circunstancias azarosas que les rodean" (ibid.: 123).

- Es una estructura de apoyo a la empleabilidad, porque ofrece oportunidades para que la persona se aproxime a las metas deseadas y favorece la conciencia de los propios avances hacia esas metas. También estructura en torno a él otros recursos que tendrá que gestionar el trabajador de inserción, con el apoyo del trabajador acompañante $u$ otras personas.

- Pertenece a la persona en inserción, que es la máxima responsable del proceso. Para ello, se requiere tanto una toma de conciencia del papel activo y protagonista de la propia persona como una sólida fundamentación en las fortalezas de las que dispone.

Los puntos que ha de recoger el plan de trabajo son típicamente: la situación del trabajador en relación al mercado laboral; los objetivos que se propone para alcanzar sus metas profesionales; la elaboración de acciones, responsabilidades, cronogramas y pautas dirigidos a la consecución de los anteriores objetivos; los apoyos necesarios; y el establecimiento del seguimiento del itinerario. El diseño de este plan de trabajo se sitúa como una especie de 'gozne' sobre el 
cual gira esta fase y la siguiente: podemos considerarlo, por ello, tanto como el indicador del final de la fase de acogida como del inicio de la de seguimiento.

\subsection{Segunda fase: seguimiento (o fase de permanencia y acompañamiento en la empresa de inserción)}

La de seguimiento es la fase de mayor duración, cuyo objetivo principal se podría resumir en 'acompañar' en el desempeño de una persona en un puesto de trabajo real. Ese acompañar conlleva implícitamente objetivos de capacitación en las áreas laboral, personal y social, tal y como se refleja en el Cuadro 1. Los avances y dificultades en el logro de estos objetivos por parte del trabajador se registran con cierta periodicidad (por ejemplo, mensual) y se contrastan con el trabajador acompañante, el técnico de producción y otros profesionales. Es en esta fase cuando se va conociendo verdaderamente a la persona en inserción (incluso aparecen datos nuevos significativos, no recogidos en la valoración inicial) y cuando surgen las cuestiones críticas relevantes de cara a la permanencia del trabajador en la empresa de inserción y a su posterior inserción en el mercado laboral ordinario.

Se trata, por tanto, de un proceso continuo de evaluación de la actuación en el puesto desempeñado y de revisión y mejora del plan de trabajo trazado, un proceso en el que debe participar activamente el propio trabajador de inserción. Es importante que, en esta revisión, no se desresponsabilice a la persona en inserción en favor de los propios prejuicios del educador que 'sí sabe lo que es mejor para ella'. Como nos advierte Orteu (2007: 65), “lo importante no será tanto avanzar con 'elecciones ganadoras', sino avanzar en cómo cada sujeto puede hacerse cargo de su futuro al interrogarse sobre su propio recorrido, es decir, hacer que sus elecciones sean ganadoras".
1.3. Tercera fase: transición al mercado laboral ordinario (o fase de finalización y apoyo a la inserción sociolaboral)

El planteamiento que se efectúa a cada trabajador desde el momento en que entra a formar parte de una empresa de inserción es el de orientarlo 'hacia fuera' durante todo el proceso, es decir, hacia su incorporación en una empresa en el mercado laboral ordinario, ya que se incide en que la empresa de inserción es una empresa de tránsito no finalista. Resulta casi imposible fijar qué plazo temporal asegura esta salida con un grado de desarrollo de empleabilidad suficiente para acceder al mercado laboral ordinario, dadas las dificultades de partida y la enorme heterogeneidad de las personas que se incorporan a una empresa de inserción. La normativa legal estatal y autonómica, no obstante, ya marca el plazo máximo que un trabajador puede permanecer en proceso de inserción (en general, dos años; excepcionalmente, un tercero). Lo que sí está claro es que no puede considerarse un logro o ‘éxito’ para una empresa de inserción el que los trabajadores de inserción 'se resistan' a salir de ella cuando ya hayan alcanzado gran parte de los objetivos acordados en el plan de trabajo y se evalúe que el proceso de inserción ha terminado. Ello exige revisar las expectativas y competencias iniciales, y compararlas con la representación que el trabajador hace de su situación previa a la salida de la empresa.

Con vistas a una óptima finalización del proceso en esta fase, se destacan tres elementos que vendrían a reforzarla:

- La permanencia como trabajador acompañante de un antiguo trabajador de inserción que actúe de referente para otras personas que se encuentran en el proceso de inserción.

\begin{tabular}{|c|c|}
\hline $\begin{array}{l}\text { Área laboral (o } \\
\text { técnico-profesional) }\end{array}$ & $\begin{array}{l}\text { - Desarrollar actitudes positivas hacia el aprendizaje y el empleo. } \\
\text { - Adquirir conocimientos y habilidades profesionales. } \\
\text { - Desempeñar tareas profesionales. } \\
\text { - Implicarse en su puesto de trabajo, incrementando los niveles de responsabilidad y productividad. } \\
\text { - Asumir normas. } \\
\text { - Emplear adecuadamente el tiempo de trabajo, cumpliendo con el horario establecido. } \\
\text { - Conocer y hacer un buen uso de sus derechos y obligaciones como trabajador. }\end{array}$ \\
\hline $\begin{array}{l}\text { Área } \\
\text { personal }\end{array}$ & $\begin{array}{l}\text { - Participar constructivamente en la dinámica de la empresa, mostrando conductas deseables y actitudes positivas. } \\
\text { - Desarrollar capacidad de iniciativa y otras cualidades de autonomía personal. } \\
\text { - Interiorizar logros y avances, con el consiguiente aumento de la propia autoestima personal. } \\
\text { - Aprender a resolver problemas de forma constructiva. } \\
\text { - Ser capaz de expresar sentimientos y problemas personales. } \\
\text { - Aumentar la capacidad crítica. }\end{array}$ \\
\hline $\begin{array}{l}\text { Área } \\
\text { social }\end{array}$ & $\begin{array}{l}\text { - Aceptar a los compañeros de trabajo y demás personal de la empresa, estableciendo relaciones positivas con } \\
\text { ellos. } \\
\text { - Crear redes de relación y apoyo social. } \\
\text { - Aprender a negociar, colaborar y participar. } \\
\text { - Aprender a analizar problemas del entorno laboral para transferir lo aprendido a la vida cotidiana. } \\
\text { - Aprender a comunicarse con otros de modo constructivo. } \\
\text { - Facilitar el conocimiento y uso de los recursos sociales, formativos y laborales que favorecen su integración posi- } \\
\text { tiva con el entorno. }\end{array}$ \\
\hline
\end{tabular}


- El trabajo en red de todas las entidades implicadas (asociaciones, servicios sociales, empresas ordinarias) para actuar en común y aprovechar el potencial formativo de una empresa de inserción. Muchas veces se da esta colaboración, pero de manera bastante informal. Se perciben dificultades para que una de estas empresas transmita bien sus objetivos y sea posible el trabajo conjunto, especialmente durante la fase de seguimiento. En esta fase muchas entidades derivantes parecen 'desentenderse' del trabajador. Tampoco resulta fácil encontrar empresas ordinarias que 'se vinculen' de manera más o menos estable con una empresa de inserción y a las que los trabajadores en inserción puedan percibir como posibilidad futura y real.

- El seguimiento en el puesto de trabajo, al menos durante seis meses. Según González y Marhuenda (2008), éste es un elemento primordial que puede asegurar enormemente una incorporación exitosa a las empresas de carácter ordinario. Durante este periodo el trabajador acompañante, junto con el encargado en la empresa ordinaria, siempre y cuando resulte posible y adecuado, propondrán nuevas acciones para afianzar el proceso de incorporación y adaptación al nuevo puesto de trabajo.

Para el cumplimiento de los objetivos marcados en cada fase, las empresas de inserción, si están calificadas como tales, tienen obligaciones que deben comunicar, acreditar y justificar. La Ley 44/2007, Ley de Empresas de Inserción, dicta las directrices generales para el cumplimiento de dichos objetivos, que deben concretarse en cada autonomía en función de su idiosincrasia. El País Vasco es una de las comunidades autónomas con mayor desarrollo normativo en este ámbito. Cabe señalar, también, que las disposiciones vigentes muestran la preocupación por las intervenciones de calidad en el apoyo, el seguimiento y el acompañamiento social para el fomento de hábitos sociales y de trabajo que propicien la inclusión o el mantenimiento en el mercado laboral ordinario de las personas en riesgo de exclusión laboral.

\section{El marco normativo de la evaluación en las empresas de inserción del País Vasco}

El desarrollo de las disposiciones vigentes sobre las empresas de inserción y su función social muestra la importancia otorgada a este tipo de empresas y la preocupación por la inserción sociolaboral ${ }^{2}$ en el País Vasco. De hecho, parten de la consideración de que las empresas de inserción son "agentes activos de la inserción sociolaboral" y que tienen el objetivo de "lograr la integración de estas personas al mercado ordinario" (Decreto 182/2008, de 11 de noviembre). Es por ello por lo que se estipulan las siguientes obligaciones derivadas de la calificación de empresa de inserción. En primer lugar, presentar anualmente,

${ }^{1}$ En la bibliografía se pueden consultar las disposiciones legales básicas sobre las que nos basamos en este apartado. antes de iniciarse el año, el plan de actividades que se va a desarrollar y, posteriormente, una memoria de actividades y de evaluación de las intervenciones realizadas. En segundo lugar, deben llevar a cabo medidas personalizadas de apoyo a la inserción sociolaboral y, en concreto, fomentar el aprendizaje de una profesión y las competencias demandadas en el mercado laboral ordinario. Junto a ello, se considera función de la empresa de inserción el acompañamiento social de las personas en riesgo de exclusión para ayudar a resolver problemas personales o derivados de las circunstancias personales que dificultan el proceso de inserción laboral. En todo este proceso, se establece la necesidad de que las empresas de inserción colaboren con los servicios públicos de empleo y los servicios sociales de base; es más, se concreta que son estos servicios públicos los que deben llevar a cabo el diagnóstico de la persona en riesgo de exclusión social y los que determinan, de acuerdo con las empresas de inserción, los criterios básicos de los itinerarios de inserción y de los convenios de inserción.

Este convenio concreta el itinerario de inserción sociolaboral a partir de las necesidades de la persona en cuestión. Es por ello por lo que incluye, por un lado, el resultado del diagnóstico y, por otro, los objetivos y resultados propuestos, la planificación y programación de la intervención -realizada por entidades públicas o privadas-, y la evaluación y seguimiento de la persona y del itinerario. Cabe señalar que la normativa relativa al convenio de inserción concreta, por un lado, el tipo de acciones que se han de desarrollar y que forman parte del itinerario $y$, por otro, las medidas de intervención y los servicios de acompañamiento.

Por lo que respecta a las acciones que se incluyen en el itinerario de inserción, las disposiciones vigentes hacen una propuesta que se caracteriza, sobre todo, por intervenir directamente en la persona. Así, por ejemplo, se explicitan como acciones que se han de llevar a cabo: a) las que promueven la estabilidad personal, la participación social y el empoderamiento; b) las relacionadas con hábitos de vida saludable, como la desintoxicación y deshabituación; c) las que aumenten el nivel formativo y las competencias para el aprendizaje a lo largo de la vida; d) las acciones para el aprendizaje de estrategias y técnicas de búsqueda de empleo o de emprendimiento; $y$ e) de forma más general, se contempla la posibilidad de sugerir acciones que promuevan la autonomía, la libertad y el desarrollo personal, y que faciliten el ejercicio de los derechos sociales, o que pudieran considerarse necesarias para garantizar la inserción social.

Junto a las acciones dirigidas a la persona, los convenios también pueden proponer algunas relativas a la mejora de las circunstancias personales. Así, por ejemplo, se indica la posibilidad de proponer acciones que faciliten el acceso a y el mantenimiento en una vivienda, o el alojamiento en condiciones adecuadas, acciones que busquen el acceso al sistema general de salud, y que faciliten la atención 
y el cuidado necesario a las personas dependientes de los destinatarios. Explícitamente, se indica que no se pueden incluir las acciones de naturaleza laboral que no se sustenten en el contrato de trabajo, en un contrato societario como socio de trabajo o socio trabajador de cooperativas, o que se desarrollen como trabajador autónomo.

Una vez firmado el convenio de inserción por la administración pública y el trabajador de inserción, se establece el inicio de las medidas de intervención y acompañamiento. Éstas son conceptualizadas como "el conjunto de servicios, prestaciones, acciones de orientación, tutoría y procesos personalizados y asistidos de trabajo remunerado, formación en el puesto de trabajo, habituación laboral y social encaminados a satisfacer o resolver problemáticas específicas derivadas de la situación de exclusión que dificultan a la persona un normal desarrollo de su itinerario en la empresa de inserción" (Decreto 182/2008, de 11 de noviembre, art. 6). Dicha función es compartida por los servicios sociales de base y la empresa de inserción, ya que se establece que estos servicios públicos deben prestar servicios de acompañamiento social directamente, o delegar la responsabilidad en la empresa de inserción o a la entidad promotora. Junto a ello, los servicios públicos deben hacer un seguimiento del itinerario en las empresas de inserción, por lo que se requiere cooperación y coordinación entre ambos agentes sociales, así como el desarrollo de los instrumentos de valoración, ya que en el País Vasco la responsabilidad es compartida entre los servicios públicos y la empresa de inserción como entidad privada.

\section{Las herramientas de valoración en las diferentes fases del itinerario en las empresas de inserción}

En un trabajo anterior publicado en esta misma revista (Llinares, Córdoba y Zacarés, 2012), se describe el proceso de obtención de los 249 instrumentos de evaluación de las empresas de inserción de todo el Estado español que fueron analizados para nuestro estudio. Cada una de las herramientas recogidas se enmarcó en una de las fases generales del itinerario de inserción. Se observa, en general, que aparecen mayor cantidad de instrumentos en las fases de acogida y seguimiento que en la final. Sin embargo, esto es dependiente del tipo de instrumento utilizado. Según su cometido, distinguimos dos tipos de herramientas durante el proceso que sigue el trabajador en la empresa. Podemos hablar de documentos administrativos y de trabajo. Los documentos administrativos se refieren a instrumentos de trámite requeridos por los servicios sociales o de empleo. En la fase de acogida, dichos instrumentos tienen como objetivo servir de cauce a los requerimientos legales necesarios para la coordinación entre la empresa y el organismo regulador, de modo que se utilizan documentos como autorizaciones, relaciones de usuarios de la empresa o acredi- taciones de la situación de exclusión. En la fase de seguimiento, se trata de informes sobre alguna situación destacable por parte del trabajador, como una sanción que necesita ser informada a los servicios sociales. En la fase final, son documentos informativos sobre una situación de baja laboral o informes de derivación a la empresa ordinaria.

Por su parte, los documentos de trabajo son los que utiliza la empresa para la recogida de información del trabajador necesaria en cada fase. Así, en la fase de acogida los instrumentos de trabajo recogen datos sociodemográficos y la evaluación inicial más o menos pormenorizada de sus características personales, familiares, académicas y laborales. En la fase de seguimiento, además de los acuerdos de inserción entre la empresa y el trabajador, se solicitan partes de asistencia a las actividades planificadas, fichas de evaluación del seguimiento del proceso de inserción, informes de actividades específicas, planificaciones de las labores semanales o mensuales, o programas individuales de inserción. En la fase final, los instrumentos recogen una evaluación del grado de empleabilidad que sirva para valorar el itinerario de inserción y técnicas para la búsqueda de empleo como apoyo a la incorporación del trabajador a la empresa ordinaria.

En el Gráfico 1 se observa un mayor número de documentos administrativos en la fase de acogida con respecto al resto, puesto que es la fase donde existe mayor cantidad de requerimientos legales por parte de los servicios sociales o de empleo, y se solicitan más instrumentos técnicos previamente tipificados y estandarizados.

Se presentan los instrumentos de evaluación de naturaleza técnica asociados a cada fase del proceso, agrupados por categorías tanto de finalidad como de frecuencia, y un análisis del panorama general que emerge en dicha fase en cuanto a las herramientas de valoración empleadas. En cada fase, destacaremos también los porcentajes comparativos para el caso de las empresas del País Vasco. Los recogemos a modo ilustrativo, no con intención representativa, dado que el número de empresas del País Vasco que se incluyeron en el estudio fue reducido, únicamente siete, menos de una cuarta parte de las existentes (Veciana, 2007).

\subsection{Fase de acogida}

En la Tabla 1 se muestran los instrumentos de trabajo, de carácter técnico, correspondientes a la fase de acogida y evaluación inicial. Se puede inferir claramente que existen tres instrumentos de trabajo bien establecidos en esta fase, al menos en cuanto a su perfil general aparente: a) un instrumento para la evaluación o diagnóstico de los factores de empleabilidad; b) un documento en el que, a modo de ficha, se recogen los datos sociolaborales y sociopersonales más relevantes de la persona evaluada; y c) un guion de entrevista sobre la historia de vida laboral y las motivaciones

(1) 

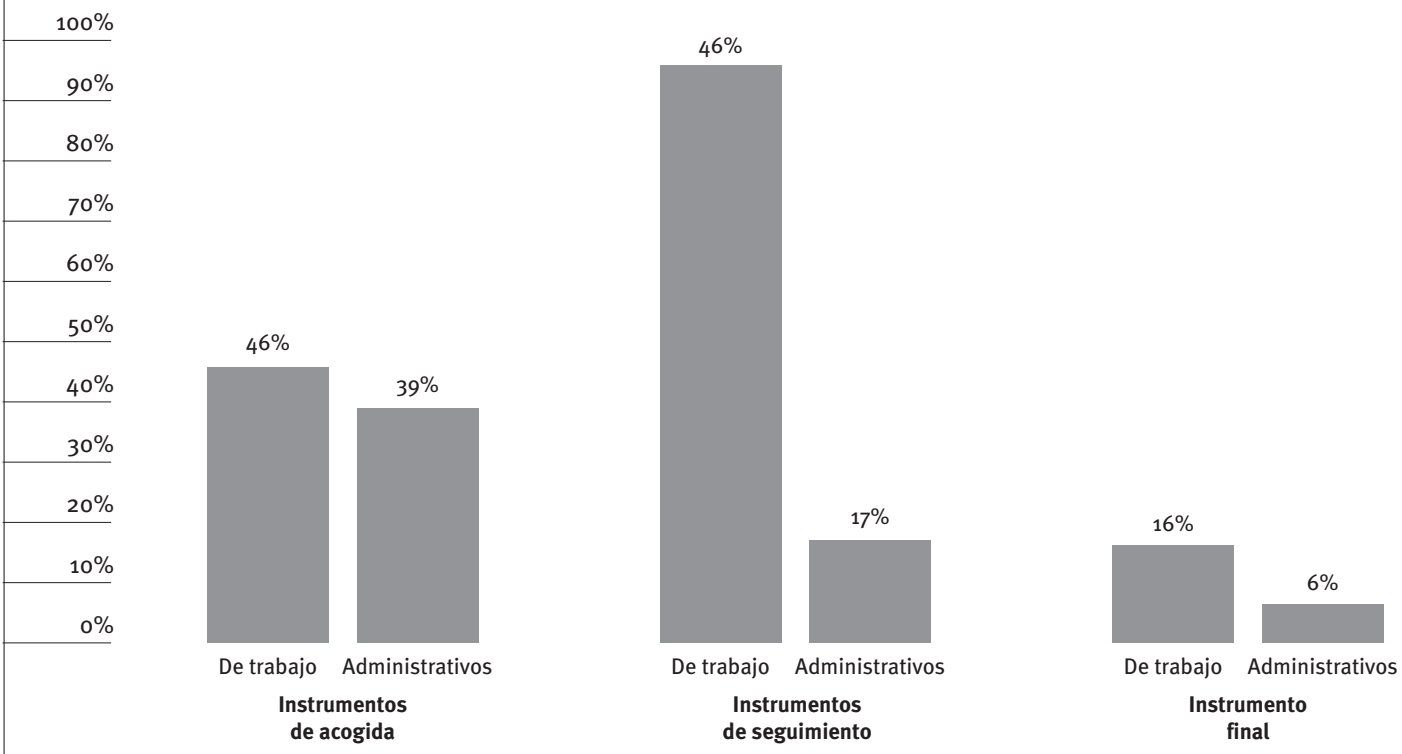

Fuente: Elaboración propia.

de entrada en la empresa de inserción. Se constata también una gran diversidad respecto al formato más o menos estructurado de las posibles respuestas a las cuestiones planteadas en estos instrumentos: se combinan desde preguntas totalmente abiertas (por ejemplo, “¿Por qué busca trabajo?”) hasta otras con unas pocas alternativas ya cerradas (por ejemplo, "Tu entorno familiar, ¿te anima buscar un trabajo?”, admitiéndose sólo como respuesta un "Sí/No").

Menos evidente resulta la diferenciación de estos tres instrumentos cuando se analizan con más detalle sus contenidos. Algunas empresas aplican los tres instrumentos por separado, mientras que otras integran las tres herramientas (diagnóstico de empleabilidad, ficha de usuario y entrevista inicial) bajo un único instrumento y una de las tres 'etiquetas’ Este hecho provoca una considerable variación en la extensión de un instrumento particular, según los objetivos que pretenda abarcar: un diagnóstico de empleabilidad puede alcanzar así las veinte hojas, con cuestiones y preguntas de amplio rango, o ser un cuestionario de dos folios, ceñido a indicadores individuales de tipo sociolaboral.

Tabla 1. Instrumentos de trabajo utilizados por las empresas de inserción durante la fase de acogida, según su frecuencia de uso (\%)

\begin{tabular}{|c|c|c|c|}
\hline Frecuencia & $\begin{array}{l}\text { Instrumento } \\
\text { (y denominaciones alternativas) }\end{array}$ & Total (\%) & País Vasco (\%) \\
\hline \multirow{3}{*}{$\begin{array}{l}\text { Alta } \\
(\leq 40 \% \text { de las } \\
\text { empresas de } \\
\text { inserción })\end{array}$} & $\begin{array}{l}\text { Diagnóstico de empleabilidad } \\
\text { (evaluación inicial del grado de empleabilidad, perfil competencial, } \\
\text { diagnóstico de ocupabilidad) }\end{array}$ & 71,4 & 42,6 \\
\hline & $\begin{array}{l}\text { Ficha de datos del usuario } \\
\text { (informe o ficha social, ficha de diagnóstico y evaluación, ficha de } \\
\text { diagnóstico de necesidades) }\end{array}$ & 64,0 & 28,3 \\
\hline & $\begin{array}{l}\text { Entrevista de acogida } \\
\text { (primera entrevista, entrevista de selección, entrevista personal } \\
\text { inicial, entrevista de nueva demanda, historial sociolaboral } \\
\text { individualizado) }\end{array}$ & 45,0 & 14,2 \\
\hline \multirow{4}{*}{$\begin{array}{l}\text { Baja } \\
(\leq 10 \% \text { de las } \\
\text { empresas de } \\
\text { inserción) }\end{array}$} & Autoevaluación ocupacional & 7,1 & 0,0 \\
\hline & Análisis de expectativas laborales & 3,6 & 14,2 \\
\hline & Baremo de candidatos & 3,6 & 0,0 \\
\hline & Listado de objetivos propuestos y de consecución & 3,6 & 0,0 \\
\hline
\end{tabular}

Fuente: Elaboración propia. 
Especialmente en el caso de que una empresa de inserción aplique los tres instrumentos separados, se llega a solicitar información muy similar en cada uno de ellos, con lo que esto tiene de redundancia y solapamiento. Un ejemplo puede ilustrar esta situación:

- En el diagnóstico de ocupabilidad, se le pregunta a la persona por los tipos de tareas que puede realizar (por ejemplo, carga y descarga, o manipulación de objetos).

- En la ficha de usuario, se le pregunta por su formación no reglada y su experiencia laboral y profesional.

- En la entrevista inicial, se indaga, mediante preguntas abiertas, sobre sus conocimientos y experiencias en diferentes áreas de trabajo (textil o reciclaje, entre otras).

Los otros instrumentos de trabajo de la Tabla 1, mucho menos frecuentes, tienen un carácter secundario y complementario, si exceptuamos la 'autoevaluación ocupacional'. Esta herramienta permite captar de un modo más directo y sencillo la autopercepción que la persona candidata a la empresa de inserción tiene de su actual situación sociolaboral, sirviendo como control de los sesgos o prejuicios que pudiera mostrar el entrevistador a la hora de interpretar las respuestas.

Podemos preguntarnos en este punto sobre el grado en que los instrumentos analizados sirven a los objetivos de esta primera fase del itinerario de inserción. Consideraremos tres aspectos que ayudan a que cualquier empresa de inserción pueda responderse a esta cuestión:

- La cantidad de información que se recoge en la evaluación inicial es considerable y supone un coste, en términos de tiempo y esfuerzo, para el evaluador y para el trabajador de inserción. Esta información es de tres tipos: a) referida a capacidades y habilidades directamente conectadas con los aprendizajes que va a fomentar la empresa de inserción (por ejemplo, habilidades personales o profesionales específicas); b) referida al contexto sociopersonal y familiar de la persona, que nos permiten 'ubicarla' psicosocialmente más allá del ámbito de intervención directa de la empresa de inserción (por ejemplo, problemas médicos, familiares o de vivienda); y c) referida a aspectos complementarios, pero irrelevantes para el proceso de inserción en esa empresa de inserción específica y que, por tanto, ya no se vuelve a utilizar a lo largo del itinerario. En principio, se esperaría que, en los instrumentos aplicados en la fase de acogida, este tercer tipo de información fuera mínima, en aras de una eficacia evaluativa. ¿Ocurre realmente así? ¿Qué porcentaje de la información recogida inicialmente se utiliza a lo largo del proceso de acompañamiento del trabajador de inserción?

- La redundancia de la información que se recoge, sobre todo cuando se utilizan varios instrumentos equivalentes en la fase de acogida. Hemos comprobado que con frecuencia se solicita del posible trabajador una información muy similar mediante diferentes herramientas. ¿Se solicita idéntica información a través de diferentes vías? Si así fuera, ¿responde ello a algún propósito que sirva para mejorar la adaptación del trabajador a la empresa? La pregunta complementaria es si hay información que sí se precisa obtener, pero que queda sin recoger, al menos con los instrumentos ya estandarizados de la empresa de inserción. Se confía entonces en que acabará siendo reunida mediante procedimientos menos sistematizados e informales.

- La representación que el trabajador se hace de su propia situación. Uno de los objetivos de la valoración inicial es, precisamente, llegar a captar la propia percepción que la persona evaluada hace de las causas de su actual situación, de las expectativas que muestra hacia su inmediato futuro y de la disponibilidad 'real' que tiene para incorporarse a la empresa de inserción. Estos aspectos pueden valorarse a través de dos opciones no excluyentes: a) permitiendo que el trabajador pueda explicar en sus propios términos las atribuciones que realiza de su propia trayectoria sociolaboral hasta el momento y de su proyección futura; b) utilizando una serie de indicadores de autoevaluación 'críticos y contrastados' de la actitud, los puntos fuertes y los puntos débiles desde los que afronta la búsqueda de trabajo en general y la incorporación a la empresa de inserción en particular. Por ejemplo, uno de los instrumentos analizados, bajo el epígrafe de 'primera entrevista', utilizaba ítems de este tipo - un total de diez-, tales como " $\mathrm{Si}$ alguien quiere localizarme por teléfono, tengo dificultades para que se pongan en contacto", “Tengo dificultades en retener o aprender lo que me dicen o enseñan" o "Creo tener claro lo que quiero, de lo que quiero trabajar".

\subsection{Fase de seguimiento}

En la Tabla 2 se muestran los instrumentos de trabajo correspondientes a la fase de seguimiento. Se constata que los tres tipos de instrumentos predominantes en ello hacen referencia a dos objetivos fundamentales:

- La descripción detallada de los compromisos de cada parte implicada en el proceso, trabajador y empresa, explicitados en un acuerdo de inserción que se irá revisando posteriormente. En dicho acuerdo aparecen especificados los objetivos y acciones que se han de desarrollar en las diferentes áreas, que responden, en general, a las tres reflejadas en el Cuadro 1: la laboral, la personal y la social.

- El seguimiento de dicho acuerdo de inserción a lo largo del periodo central en el que el trabajador de inserción permanece en la empresa de inserción. Este seguimiento se realiza de dos modos: 
Tabla 2. Instrumentos de trabajo utilizados por las empresas de inserción durante la fase de seguimiento, según su frecuencia de uso (\%)

\begin{tabular}{|c|c|c|c|}
\hline Frecuencia & $\begin{array}{l}\text { Instrumento } \\
\text { (y denominaciones alternativas) }\end{array}$ & Total (\%) & País Vasco (\%) \\
\hline \multirow{3}{*}{$\begin{array}{l}\text { Alta } \\
(\leq 40 \% \text { de las } \\
\text { empresas de } \\
\text { inserción) }\end{array}$} & $\begin{array}{l}\text { Evolución de la situación personal en las distintas áreas } \\
\text { (valoración del seguimiento en el programa individualizado, hoja } \\
\text { de seguimiento del plan de trabajo, evaluación del convenio de } \\
\text { inserción, seguimiento organizado por competencias) }\end{array}$ & 70,0 & 42,6 \\
\hline & $\begin{array}{l}\text { Acuerdo de inserción } \\
\text { (plan individual de inserción, contrato de inserción sociolaboral, } \\
\text { itinerario personalizado de inserción sociolaboral, plan de actuación } \\
\text { personalizado inicial) }\end{array}$ & 64,3 & 28,4 \\
\hline & $\begin{array}{l}\text { Ficha de seguimiento del trabajador en el puesto de trabajo } \\
\text { (ficha de desempeño mensual, seguimiento orientación laboral, } \\
\text { ficha de seguimiento individual) }\end{array}$ & 43,0 & 28,4 \\
\hline \multirow{5}{*}{$\begin{array}{l}\text { Media } \\
\text { (15-30\% de las } \\
\text { empresas de inserción } \\
\text { social) }\end{array}$} & Compromisos del itinerario de inserción & 32,0 & 0,0 \\
\hline & $\begin{array}{l}\text { Resumen mensual de intervención } \\
\text { (ficha mensual de evaluación general) }\end{array}$ & 25,0 & 14,2 \\
\hline & $\begin{array}{l}\text { Evaluación del seguimiento del grado de empleabilidad } \\
\text { (evaluación intermedia del perfil competencial, capacidades clave } \\
\text { del perfil profesional) }\end{array}$ & 21,4 & 14,2 \\
\hline & Control de asistencia a tutorías individualizadas & 18,0 & 0,0 \\
\hline & $\begin{array}{l}\text { Diario del acompañante laboral } \\
\text { (supervisión de competencias del trabajador de empresa de } \\
\text { inserción social, ficha de observaciones) }\end{array}$ & 18,0 & 0,0 \\
\hline \multirow{13}{*}{$\begin{array}{l}\text { Baja } \\
(\leq 10 \% \text { de las } \\
\text { empresas de } \\
\text { inserción) }\end{array}$} & Valoración trimestral de cumplimiento de objetivos & 7,1 & 14,2 \\
\hline & $\begin{array}{l}\text { Seguimiento del trabajo realizado } \\
\text { (informe sobre productividad en el puesto de trabajo) }\end{array}$ & 7,1 & 14,2 \\
\hline & Indicadores de seguimiento para acción formativa & 7,1 & 0,0 \\
\hline & Resumen semanal de asistencia & 3,6 & 0,0 \\
\hline & Convocatoria y acta de reunión de seguimiento & 3,6 & 0,0 \\
\hline & Hoja de seguimiento quincenal & 3,6 & 0,0 \\
\hline & Parte de asistencia a acciones grupales & 3,6 & 0,0 \\
\hline & Solicitud de intervención & 3,6 & 0,0 \\
\hline & Descripción del organigrama y del puesto de trabajo & 3,6 & 0,0 \\
\hline & Ficha de valoración rápida & 3,6 & 0,0 \\
\hline & Compromisos de horarios y normas básicas de funcionamiento & 3,6 & 0,0 \\
\hline & Planificación del trabajo mensual & 3,6 & 0,0 \\
\hline & $\begin{array}{l}\text { Valoración del trabajador } \\
\text { (del encargado/ compañeros/otras personas) }\end{array}$ & 3,6 & 14,2 \\
\hline
\end{tabular}

Fuente: Elaboración propia.

a) una evaluación general de los objetivos marcados en cada una de las áreas con una periodicidad muy variable (semana, quincenal o mensual, por ejemplo); b) una evaluación más detallada y precisa de la ejecución y rendimiento del trabajador de inserción en el puesto de trabajo asignado, en la que se valore toda una serie de tareas y actividades laborales específicas.

Los otros instrumentos de trabajo que aparecen dos objetivos en forma de registros de asistencias, síntesis del seguimiento o planificaciones temporales. Destaca entre ellos el diario del acompañante laboral como una herramienta imprescindible para recoger múltiples observaciones del comportamiento cotidiano del trabajador en la empresa de inserción. Estas observaciones recogen, en conjunto, la evolu- ción del trabajador y elementos clave sobre aspectos por revisar y optimizar en el itinerario. Otro instrumento muy poco empleado, pero que podría resultar también de interés es el que permite que el propio trabajador de inserción evalúe a su encargado y a sus compañeros. De nuevo obtendríamos así un acceso a la autopercepción del trabajador en el itinerario, en este caso referida a sus relaciones interpersonales, a la vez que un contraste adecuado de las intervenciones efectuadas.

Los instrumentos de evaluación analizados en esta fase presentan como características generales:

- Una gran diversidad en su construcción formal y en su denominación. La forma de evaluar los objetivos acordados es bastante diferente entre las empresas de la muestra; varían las competencias 
evaluadas, así como la periodicidad y el nivel de detalle con el que se solicita la información.

- Un formato de respuesta menos cerrado y estructurado que el de los instrumentos de la fase inicial.

- Una cierta discontinuidad respecto a las áreas evaluadas en la fase de acogida, puesto que los indicadores realmente valorados en la fase de seguimiento son menos numerosos y no del todo equivalentes.

En nuestra opinión, el cumplimiento de los fines generales de esta fase exigiría de los instrumentos de evaluación una serie de condiciones para ser plenamente funcionales:

- Deberían permitir la evaluación del avance en los objetivos operativos de las tres áreas recogidos en el Cuadro 1, adaptados a las características de cada trabajador y plasmados en el acuerdo de inserción. En el área laboral, de modo específico, se precisaría una valoración más detallada y continuada de aquellas tareas asociadas al puesto de trabajo que se considerasen clave para cumplir los objetivos operativos de "adquirir conocimientos y habilidades profesionales”, “desempeñar tareas profesionales" e "implicación en su puesto de trabajo incrementando su nivel de responsabilidad y de productividad" (González y Marhuenda, 2008).

- Han de recoger la integración de diversas perspectivas en la evaluación: la del trabajador acompañante, la del técnico de producción responsable, la de otros compañeros del trabajador $y$, si es el caso, la de otras figuras profesionales que intervengan. Resulta especialmente importante asegurar la conexión entre las observaciones cotidianas e informales del trabajador acompañante y del técnico de producción con los objetivos operativos recogidos en el acuerdo de inserción: ¿se puede establecer claramente esa conexión directa? Para ello, la comunicación ha de ser fluida y constante entre los implicados.

- Han de fomentar la toma de conciencia en el trabajador de su papel protagonista en el propio itinerario de inserción. Su perspectiva particular sobre los avances y dificultades en el proceso y sobre el ritmo a seguir en él ha de estar incorporada en los instrumentos de evaluación a lo largo de toda la fase. Un requisito previo para esta toma de conciencia es que en la fase de seguimiento podamos ya verificar que el trabajador ha roto con su propia 'autoetiqueta autoexcluyente', es decir, esa autopercepción fruto de toda la historia personal previa, que confirma a los trabajadores de inserción su visión como personas excluidas socialmente. ¿Pueden contribuir a ello las herramientas de evaluación empleadas? Podrían ayudar a ello en la medida en que focalicen al trabajador sobre sus fortalezas conseguidas, sus nuevos aprendizajes y sobre una perspectiva temporal positiva, 'en avance'.
- Han de ser sensibles a las variaciones en el ritmo al que progresa la persona en inserción. De este modo, los instrumentos deben proporcionar información útil para ajustar las intervenciones y revisar el acuerdo de inserción en función de cómo se vaya produciendo la adaptación particular de cada trabajador a su entorno de trabajo y de las otras circunstancias personales concurrentes que inevitablemente van a influir en dicha adaptación. Utilizar instrumentos asociados a diversas periodicidades temporales es una buena estrategia, puesto que lo que en algunos trabajadores se puede constatar quincenalmente en otros sólo será posible cuando ampliamos el periodo temporal de comparación.

\subsection{Fase de transición al mercado laboral ordinario}

En la Tabla 3 se muestran las escasas herramientas evaluativas técnicas correspondientes a la fase de transición al mercado laboral normalizado. Se han detectado pocos instrumentos elaborados o adaptados para facilitar la transición del trabajador de inserción al mercado laboral, cuando algunos de los que aparecen en la Tabla 3 deberían estar ampliamente asumidos, como el informe final del itinerario seguido por un trabajador de inserción que incluyese una valoración socioeducativa de todo el proceso. Las razones de ello pueden ser varias. Así, en el caso de algunas empresas de inserción, los procesos de orientación y apoyo a la búsqueda de empleo pueden estar delegados en otras instancias de intermediación bien de la misma entidad, bien de entidades afines con las que se trabaja en red, con lo que la propia empresa no se ve en la necesidad de generar ninguna herramienta propia para esta fase. En otros casos, se volvería a confiar en realizar esa tarea de un modo más informal y menos sistemático, empleando el criterio experimentado y flexible del trabajador acompañante o el de otros profesionales implicados.

Lo que sí que resulta evidente es que se recoge y sintetiza poca información de la que se ha ido reuniendo a lo largo de todo el itinerario, al menos de aquella que sí podría ser útil para la orientación en esta etapa. Con esto no sólo se puede ser menos efectivo en el seguimiento del trabajador, sino lo que nos parece más importante, se pierde la oportunidad de utilizar una información 'crítica' para la autoevaluación de la propia empresa de inserción.

En efecto, con la información contenida en un informe de valoración final del itinerario que también incluyese aquellos aspectos relevantes positivos y negativos de la actuación de la empresa en el itinerario de un trabajador concreto, se podrían ir apuntando direcciones de mejora continuada en las prácticas de intervención. Podemos suponer las diversas cuestiones que surgirían en la reflexión final sobre un itinerario concreto: ¿qué hizo que este trabajador diese un giro positivo en su trayectoria en la empresa de inserción?, ¿qué pudo fallar en la 
Tabla 3. Instrumentos de trabajo utilizados por las empresas de inserción durante la fase de transición al mercado laboral ordinario

\begin{tabular}{|c|c|c|c|}
\hline Frecuencia & $\begin{array}{l}\text { Instrumento } \\
\text { (y denominaciones alternativas) }\end{array}$ & Total (\%) & País Vasco (\%) \\
\hline \multirow{10}{*}{$\begin{array}{l}\text { Baja } \\
(\leq 10 \% \text { de las } \\
\text { empresas de } \\
\text { inserción) }\end{array}$} & $\begin{array}{l}\text { Agenda de búsqueda de empleo } \\
\text { (herramientas para organizar la búsqueda de trabajo) }\end{array}$ & 10,0 & 14,2 \\
\hline & $\begin{array}{l}\text { Informe laboral de acompañamiento a la derivación } \\
\text { (memoria de intervención, evaluación final del proceso en la } \\
\text { empresa de inserción social) }\end{array}$ & 10,0 & 0,0 \\
\hline & $\begin{array}{l}\text { Valoración final del itinerario } \\
\text { (informe socioeducativo final, información a los recursos de origen } \\
\text { al finalizar la fase formativa) }\end{array}$ & 10,0 & 14,2 \\
\hline & $\begin{array}{l}\text { Medición de satisfacción en la empresa de inserción social } \\
\text { (cuestionario de satisfacción con el servicio, valoración final de los } \\
\text { usuarios) }\end{array}$ & 10,0 & 0,0 \\
\hline & Evaluación final del grado de empleabilidad & 7,1 & 0,0 \\
\hline & Planificación semanal de búsqueda de empleo & 3,6 & 0,0 \\
\hline & Ficha de gestión de ofertas & 3,6 & 0,0 \\
\hline & Informe proceso búsqueda activa de empleo & 3,6 & 0,0 \\
\hline & Ficha de empresa & 3,6 & 0,0 \\
\hline & Control de inserción & 3,6 & 0,0 \\
\hline
\end{tabular}

Fuente: Elaboración propia.

evaluación inicial de un trabajador para haber dejado fuera una información personal que tuvo luego gran importancia?

\section{Conclusiones}

El análisis por fases de una muestra representativa de los instrumentos de evaluación que emplean las empresas de inserción nos lleva a plantear unas conclusiones finales sobre el grado en que las herramientas de evaluación están sirviendo a los fines generales de toda empresa de inserción. Presentamos a continuación estas conclusiones en forma de retos pendientes y sugerencias de mejora para la acción evaluativa.

\subsection{Validez ecológica de los instrumentos de evaluación}

En los últimos años existen ya a disposición de las empresas de inserción diversos instrumentos y herramientas estandarizados derivados de modelos generales sobre los factores que inciden en la empleabilidad (por ejemplo, Ballester, Caballer y Hernández, 2008; Fernández, Galarreta y Martínez, 2007; Orteu, Pi y Poch, 2007). Es una práctica habitual que, a partir de aquellos instrumentos y con leves modificaciones, se difunda su aplicación entre entidades que no los elaboraron ni experimentaron, a la vez que se va perdiendo la fundamentación y sentido originales de los indicadores, preguntas y escalas contenidos en dichos instrumentos. Éste es, en nuestra opinión, un primer reto: garantizar la 'validez ecológica' de los instrumentos empleados, es decir, conseguir que resulten altamente contextualizados y ajustados a las características y necesidades de la empresa de inser- ción. También han de tener en cuenta los recursos, ayudas y programas existentes.

Cada empresa de inserción ha de preguntarse, en un análisis previo, sobre el tipo de información que realmente necesita en cada fase del itinerario para asegurar una máxima eficacia evaluativa. Las características psicosociales de los colectivos con los que se trabaje habitualmente, el sector productivo al que se dedique, con sus perfiles asociados de competencias profesionales y las especificidades socioeconómicas del entorno geográfico donde se halle ubicada son tres de los elementos que, como mínimo, se han de introducir en la reflexión. Otras cuestiones particulares relativas a la organización y gestión de los recursos humanos y de las tareas en cada empresa de inserción podrían asimismo añadirse.

\subsection{Simplificación y relevancia de los instrumentos e indicadores recogidos}

Tras identificar los núcleos de datos e informaciones que resultan 'críticos' y relevantes, el siguiente reto es generar herramientas más simplificadas y reducidas en número, pero más significativas. Sugerimos algunas vías exploratorias en este sentido:

- ¿Se pueden unificar instrumentos en algunas de las fases? Resulta bastante factible esa unificación para el caso del instrumento de recogida de información inicial, que asumiría esa función básica de 'evaluación de empleabilidad'. Fernández et al. (2007) presentan un buen ejemplo de esta herramienta unificadora, la 'ficha de acceso', que contiene siete apartados: datos de identificación, situación personal, historia profesional, competencias, expectativas profesionales, documentos 
asociados y orientación inicial. También se puede adoptar un único instrumento de síntesis que evalúe, en la fase de transición al mercado de trabajo ordinario, los puntos fuertes y débiles del itinerario recorrido por un trabajador concreto.

- ¿Puede ser útil diseñar instrumentos semiestructurados que recojan las respuestas y datos del trabajador mediante una serie de alternativas que el contraste continuado con la realidad ha confirmado son las más frecuentes y relevantes? Un ejemplo de ello nos lo proporciona uno de los guiones de entrevista inicial analizados. Ante la pregunta de “¿Qué crees que te aportaría un trabajo?", se ofrece una síntesis de los motivos principales que podría alegar el candidato a incorporarse a la empresa de inserción: "ingresos estables / acceder a una vivienda / organizar el tiempo diario / sentirse realizado personalmente / colaborar en la economía familiar / regularizar la situación legal”.

- ¿Resulta factible valorar más directamente la percepción del propio trabajador de sus condicionantes, disponibilidad laboral y expectativas ante el proceso de inserción? Las herramientas han de ser sensibles al punto de vista de la persona en inserción, recogiendo sus aspiraciones a lo largo de todo el itinerario. Como ya hemos descrito más arriba, también resulta posible utilizar una amplia gama de indicadores de autoinforme claros e informativos a lo largo de todas las fases.

\subsection{Coherencia e integración de la información recogida entre- e intra-fases del itinerario}

Observamos una clara diferenciación entre los instrumentos utilizados en la fase de seguimiento con respecto a los de la fase previa: cambian los formatos y los indicadores recogidos. El reto es ahora la coherencia evaluativa. Habría que salvaguardar al menos dos tipos de coherencia: una 'entre-fases' y otra 'intra-fase'. La 'entre-fases' hace referencia a la coherencia entre el resultado de la valoración inicial y los objetivos planteados en el itinerario individualizado de inserción. La coherencia 'intra-fase' posee una doble vertiente: por un lado, se buscaría la conexión entre los objetivos del acuerdo de inserción y las herramientas de evaluación del seguimiento en las diferentes áreas; por otro lado, se tendrían que vincular las observaciones continuadas del trabajador acompañante, del técnico de producción y de los compañeros de trabajo sobre el desempeño profesional con el avance en dichos objetivos. ¿Cómo lograr incrementar esta coherencia?

Una posibilidad poco explorada es hacer uso de instrumentos muy similares a lo largo de cada una de las fases que constaten los cambios observados en el trabajador y la consecución de los objetivos pro- puestos. Hemos verificado cómo algunas empresas emplean un instrumento idéntico para evaluar el grado de empleabilidad, aplicándolo en el momento inicial, intermedio y final del itinerario. Estas herramientas 'coherentes' también han de ser 'procesuales', es decir, que permitan la recogida progresiva de información a medida que el trabajador recorre el itinerario y se adapten a las variaciones de acuerdo a los ritmos en que cada uno lo hace.

\subsection{Destinatarios de la información}

Los destinatarios de la información obtenida durante el proceso de inserción son básicamente tres: instancias externas a las empresas de inserción, la propia empresa y el trabajador de inserción. La información recogida mediante procedimientos más sistematizados, además de incluirse en los informes requeridos externa o legalmente, resulta de gran utilidad para los otros destinatarios. Para ello, los instrumentos han de satisfacer, además de las características ya mencionadas anteriormente, la de 'accesibilidad', es decir, la de ser herramientas comprensibles para todos, pues utilizan términos claros y sencillos, y evitan la tecnificación innecesaria.

Más allá de evaluar los objetivos planteados en el proyecto de inserción, la devolución en forma de síntesis de la información recogida cumple dos importantes finalidades: a) permite que la persona en inserción ‘se apropie’ más intensamente de un itinerario que le pertenece (González y Marhuenda, 2008), sea más realista en su revisión y afronte su salida de la empresa de inserción con mayores expectativas de autoeficacia; b) y permite que la propia empresa de inserción tenga una base objetiva para reflexionar sobre sus prácticas y la introducción de cambios optimizadores en ellas.

\subsection{Una reflexión final}

Somos conscientes de que las múltiples tareas que los profesionales realizan en el proceso de acompañamiento dificultan otras igualmente importantes, pero menos 'inmediatas', como podría ser la de atender a la dimensión evaluativa. Sin embargo, si no se afrontan periódicamente estas tareas, el funcionamiento cotidiano de la empresa de inserción también acaba resintiéndose, cumpliendo sus objetivos de modo más deficiente. Éste es un último reto, la mejora continuada en los procedimientos y herramientas de evaluación. La existencia de una normativa legal que especifica en gran medida los aspectos que se han de evaluar, tal y como sucede en el caso del País Vasco, debe complementarse con una actividad autorreflexiva y creativa de cada empresa de inserción sobre el sentido y utilidad real de sus propias herramientas de evaluación. 


\section{Referencias bibliográficas}

BALLESTER, G.; CABALLER, M.; y HERNÁNDEZ, A. (2008): Instrumentos de trabajo. Itinerarios de inserción desde la perspectiva de trabajo en red. Instrumentos y metodología, Valencia, Consorcio Pactem Nord.

ESPAÑA (2007): “Ley 44/2007, de 13 de diciembre, para la regulación del régimen de las empresas de inserción", Boletín Oficial del Estado, nํ2 299, 14-12-2007, págs. 51.331-51.339 [<http://www.boe.es/ buscar/doc.php?id=BOE-A-2007-21492〉].

FERNÁNDEZ, A.; GALARRETA, J.; y MARTÍNEZ, N. (coords.) [2007]: Manual de acompañamiento en las empresas de inserción: proceso y herramientas. Una propuesta desde la práctica, Bilbao, Proyecto Equal-Lamegi.

GONZÁLEZ, N.; y MARHUENDA, F. (2008): “El proceso de inserción social dentro de las empresas de inserción”, en GONZÁLEZ, N.; y MARHUENDA, F. (coords.), El trabajador acompañante. Nuevo perfil en las estructuras empresariales, Valencia, Carena, págs. 111-134.

LLINARES, L.; CÓRDOBA, A. I.; y ZACARÉS, J. J. (2012): “La medida de la empleabilidad en las empresas de inserción en el País Vasco: de la exclusión a la inserción sociolaboral”, Zerbitzuan, $\mathrm{n} \times 51$, págs. 83-94 ['http://dx.doi.org/10.5569/1134$7147 / 51.05>]$

ORTEU, X. (2007): La construcción de itinerarios de inserción laboral. Una propuesta metodológica, Barcelona, Gedisa.

ORTEU, X.; PI, J.; y POCH, M. (eds.) [2007]): Guía d' inserció sociolaboral adreçada als professionals de l'àmbit de les drogodependències, Barcelona, Departament de Salut de la Generalitat de Catalunya.

PAÍS VASCO (2009): “Orden de 4 de mayo de 2009, de 11 de noviembre, regula las ayudas para la creación y sostenimiento de las empresas de inserción”, Boletín Oficial del País Vasco, no 98, 26-5-12 [khttp://www.lehendakaritza. ejgv.euskadi.net/r48-bopv2/es/bopv2/ datos/2009/05/0903002a.pdfı].

- (2008): “Decreto 182/2008, de 11 de noviembre, regula la calificación de empresas de inserción, se establece el procedimiento de acceso a las mismas y su registro", Boletín Oficial del País Vasco, no 225, 24-11-2008, págs. 28.250-28.264 [<http://www. lehendakaritza.ejgv.euskadi.net/r48-bopv2/ es/bopv2/datos/2008/11/0806515a.pdf)].

- (2007): “Orden de 3 de mayo de 2007, del Consejo de Justicia, Empleo y Seguridad Social, por la que se modifica la Orden 11 de diciembre de 2002, por la que se regulan las ayudas para la creación y el sostenimiento de las empresas de inserción”, Boletín Oficial del País Vasco, 꾸 90, 11-5-2007, págs. 10.954-10.957 [khttp://www. lehendakaritza.ejgv.euskadi.net/r48-bopv2/ es/bopv2/datos/2007/05/0702665a.pdfs].

- (2000): “Decreto 1/2000, de 11 de enero, por el que se regulan los Convenios de Inserción", Boletín Oficial del País Vasco, nํ․ 20, 31-1-2000, págs. 1.800-1.815 [<http://www.lehendakaritza. ejgv.euskadi.net/r48-bopv2/es/bopv2/ datos/2000/01/0000408a.pdf〉].

- (2000): “Decreto 305/2000, de 26 de diciembre, por el que se regula la calificación de las empresas de inserción, se establece el procedimiento de acceso a las mismas y se crea el registro de empresas de inserción", Boletín Oficial del País Vasco, no2 24, 2-2-2001, págs. 1.900-1.913 [<http://www.lehendakaritza. ejgv.euskadi.net/r48-bopv2/es/bopv2/ datos/2001/02/0100592a.pdf >].

- (1998): “Ley 12/1998, de 22 de mayo, contra la Exclusión Social”, Boletín Oficial del País Vasco, nํㅜ 105, 8-6-1998, págs. 10.467-10.506 [khttp://www. lehendakaritza.ejgv.euskadi.net/r48-bopv2/ es/bopv2/datos/1998/06/9802519a.pdf)].

VECIANA, P. (coord.) [2007]: Las empresas de inserción en España, Barcelona, Fundació Un Sol Món.

ZACARÉS, J. J.; CÓRDOBA, A. I.; y LLINARES, L. I.; (2011): "Análisis de los instrumentos de valoración empleados por las empresas de inserción social. Una visión desde las fases del proceso", en CÓRDOBA, A. I. y MARTÍNEZ, I. (coords.), Trabajo, empleabilidad y vulnerabilidad social: condicionantes $y$ potencialidades de la integración a través de las empresas de inserción social, Valencia, Servicio de Publicaciones de la Universitat de València. 